\title{
The free energy of a liquid when viewed as a population of overlapping clusters
}

\author{
Pierre Ronceray \\ LPTMS, CNRS, Univ. Paris-Sud, \\ Université Paris-Saclay, 91405 Orsay, France \\ and Peter Harrowell \\ School of Chemistry, University of Sydney, Sydney N.S.W. 2006, Australia
}

\begin{abstract}
The expression of the free energy of a liquid in terms of an explicit decomposition of the particle configurations into local coordination clusters is examined. We argue that the major contribution to the entropy associated with structural fluctuations arises from the local athermal constraints imposed by the overlap of adjacent coordination shells. In the context of the recently developed Favoured Local Structure model [Soft Matt. 11, 3322 (2015) ], we derive explicit expressions for the structural energy and entropy in the high temperature limit, compare this approximation with simulation data and consider the extension of this free energy to the case of spatial inhomogeneity in the distribution of local structures.
\end{abstract}

\section{INTRODUCTION}

To distinguish crystals from liquids by the presence or absence of periodic order, while operationally sound, is a lob-sided and potentially misguided basis for the description of either material. Clearly, the absence of periodicity in the liquid tells us only what the liquid is not. As for the crystal, periodicity can indeed describe the local arrangements of particles that determine the energetics of the phase, but only for crystals with small unit cells. A more generally useful distinction between the two phases is that a crystal is structurally homogeneous while a liquid is structurally heterogeneous. In a survey of the 4264 structures of the inorganic crystal structure data base, Daams and Villars [1] found that $88 \%$ exhibited only 4 or less distinct local coordination environments. A survey [2] of molecular crystals found that $92 \%$ consisted of only a single distinct coordination site. In contrast, simulations of amorphous binary alloys regularly report between 15 and 20 distinct local coordination polyhedra occurring with significant frequency. This number can only increase in the 3 to 5 component alloys frequently used in bulk metallic glasses. An approach to liquids based on the multiple possible local coordination structures would, by default, also cover the crystal structures. This consistent representation of the structure of both condensed phases provides one the fundamental motivations for the subject of this paper.

To refer to an arrangement of particles as a structure is to imply that it can be intelligibly decomposed into some finite number of components. A number of choices for these components have been considered - Voronoi polyhedral [3, Delaunay tessellations 4, common neighbours [5], bond ring statistics [6] and tetrahedrality [7]. One of the most common is the local coordination polyhedra 8] similar to a Delaunay vertex except that the neighbours are defined by a cutoff separation rather than a geometric condition based on space-filling. The uses of local coordination shells to construct complex extended structures is the basis of the classic work of Frank and Kasper 9. The coordination polyhedra have the appealing property that, for liquids comprised of particles that interact only by short range potentials, the potential energy is completely specified by the statistics of the coordination shells. The other attractive feature of any structural description is data compression. Indeed, the full description of the environment of a particle involves $n d$ continuous degrees of freedom, where $n$ is the number of neighbours of a site and $d$ the space dimension. In contrast, once a dictionary of distinct structures has been established, the local environment can be specified simply by indicating in which of the $N_{s}$ possible environments the particle is. Such structural characterizations, therefore, correspond to a substantial coarse-graining (or up scaling 10]). What is neglected in this description are the continuum of distortions local and global - that would effectively differentiate each local structure and result in elastic-like coupling between local structures over an extended range. The discretization of the space of structures gained through this coarse graining approximation is of considerable benefit for the calculation of the liquid free energy both in terms of the mathematical practicalities (replacing integrals by sums) and in providing some sort of intuition regarding the connection between the particle properties and the stability of their liquid.

In a recent series of paper 10 14, Procaccia and coworkers have developed an empirical approach to the construction of a free energy in terms of an explicit decomposition of liquid structures into local coordination clusters which they refer to as quasi-species. They have applied this quasi-species construction to binary atomic mixtures in 2D [11 and 3D 12, a molecular liquid 13 and a liquid characterised by tetrahedral coordination [14. As described in ref. 10, nearest neighbour clusters are categorized by the total number of particles in the coordination shell. In a binary mixture of large and small atoms, the mole fraction of clusters are then denoted as $C_{s}(n)$ and $C_{l}(n)$ where $n$ is the number of neighbours and $s$ or 
$l$ denote the identity of the central particle. The composition of the coordination shell is not included in this classification. Given the constraints of packing, there are only a finite number of values of $n$ likely to be found about a particle of a given species, so the number of types of cluster is finite $(\sim 5-6$ in $2 \mathrm{D}$ and $15-20$ in $3 \mathrm{D}$ for the size ratios studied [11, 12]). Next, the average value of the structure concentrations $\left\langle C_{\alpha}(n)\right\rangle(T)$ are calculated over a range of temperatures and then inverted to give a cluster free energy $F_{\alpha}(n, T)$ using

$$
\left\langle C_{\alpha}\right\rangle(T)=\frac{\exp \left(-F_{\alpha}(m, T) / T\right)}{2 \sum_{m} \exp \left(-F_{\alpha}(m, T) / T\right)}
$$

Note that this approach has factorized the free energy by neglecting any contribution to the free energy from interactions between clusters. The resulting free energies have been found to vary linearly with $\mathrm{T}$ over the temperature range studied, a result that permits an enthalpy and entropy to be defined for each cluster size, i.e.

$$
F_{\alpha}(n, T) \approx h_{\alpha}(n)-T s_{\alpha}(n)
$$

The quasi-species construction is essentially descriptive; a strategy for finding an optimal extrapolation of the observed $T$ dependence of the cluster concentrations. A shortcoming of this approach is the neglect of the correlations between neighbouring local structures in the calculation of the entropy. Adjacent coordination clusters must overlap, so that a specific coordination on particle will impose a constraint on the possible coordination shells of its neighbour. Such constraints are geometric and hence athermal and they result in complex correlations between the concentrations of various clusters. In this paper we present an explicit treatment of this problem in the calculation of the structural entropy. To this end, we employ a lattice model of liquids, namely the Favoured Local Structure model, which we introduce in the following Section. In Section 3 we derive an explicit expression for the liquid free energy in the high temperature limit based on an expansion in powers of the structure concentrations. A comparison between the approximate theory and Monte Carlo calculations is presented. In Section 4 we consider the extension of the treatment to address the free energy of the solid-liquid interface.

\section{THE FAVOURED LOCAL STRUCTURE MODEL}

The central challenge to developing a statistical mechanics based on local structures is to handle the identification and counting of these structures. Allowing particles to only occupy discrete positions in space i.e. considering a lattice liquid significantly simplifies these difficulties. This was a major motivation behind our development of the Favoured Local Structure (FLS) model. A lattice is selected and every vertex assigned one of two values. We shall refer to these two states as spin up or down. (In a standard mapping, the states can equivalently be thought of as atomic species A or B whose identity can be interchanged in a Monte Carlo procedure.) The local structure associated with a given site is determined by the geometrical arrangement of the spins in the nearest neighbour sites. We can enumerate the $N_{s}$ possible distinct local structure, where two structures are considered distinct if they cannot be transformed into each other by a rotation. For the $2 \mathrm{D}$ triangular lattice, $N_{s}=14$ and for the 3D face centred cubic (fcc) lattice we have $N_{s}=218$. (Note that in contrast with previous works [15 19], we distinguish here spin inverted structures.) We assign an energy $\epsilon_{i}$ to each local structure, typically selecting a small number to have negative energies and hence be 'favoured' (see Fig. 1). We shall refer to the model as the Favoured Local Structure (FLS) model. The essence of our Hamiltonian is that the energy attributed to each site is determined by the entire local structure, not by the addition of pairwise interactions. A $2 \mathrm{D}$ version of the FLS model has been studied [15] with the aim of establishing the relation between the choice of FLS and the liquid entropy [16] and the freezing transition [17. A novel liquid-liquid phase transition was identified, in 2D, when the FSL was chiral [18. The extension of the FLS model to 3D based is the subject of ref. [19. This paper includes a comprehensive survey of the groundstates and phase transitions for all possible single FLS choices on a fcc lattice. The numerical simulations of the model reported below have been carried out using the Metropolis Monte Carlo algorithm due to Bortz et al. 20, employing periodic boundary conditions on an fcc lattice of typical size $24 \times 24 \times 24$.

\section{STRUCTURAL ENTROPY: THE HIGH TEMPERATURE EXPANSION}

We now introduce a framework to study the implications of the local structures on the liquid free energy. Let $\vec{c}$ be the vector of length $N_{s}$ whose components $\left\{c_{i}\right\}$ are the concentrations of the $N_{s}$ distinct local structures. The proportion of sites with local environment $i$ is thus equal to $c_{i}$. As we include all possible environments, it follows that $\sum_{i} c_{i}=1$. At infinite temperature, all spin configurations are equiprobable, and thus the concentration vector $\vec{c}_{\infty}$ at infinite temperature is given by

$$
c_{\infty, i}=g_{i} / 2^{z}
$$

where $z$ is the coordination number of the lattice $(z=12$ on the fcc lattice). Here $g_{i}$ is the rotational degeneracy of $i$ th structure, which ranges between 1 (for the all-up and all-down structures) and 24 (for any of the 136 minimally symmetric structures). For convenience we define the shifted concentrations

$$
\vec{\phi}=\vec{c}-\vec{c}_{\infty}
$$



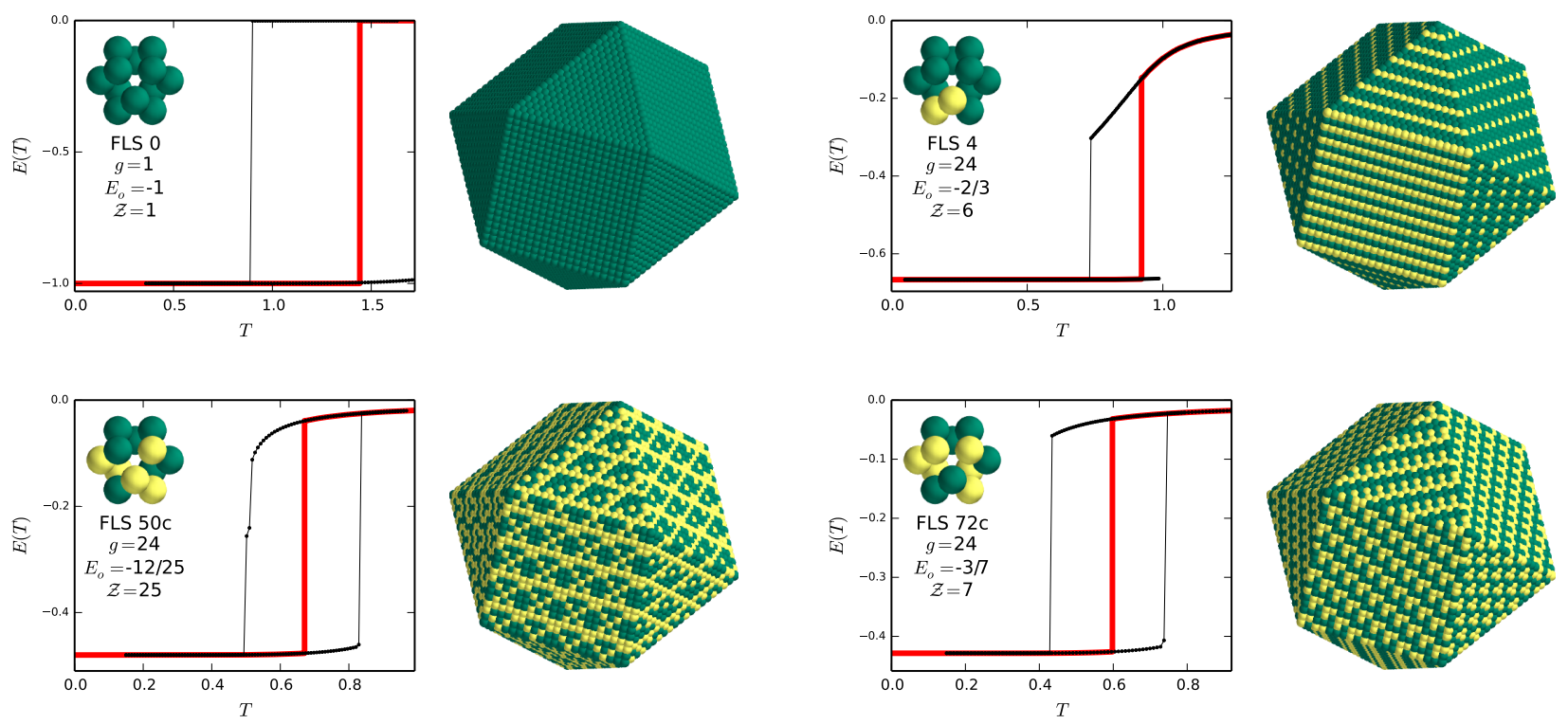

FIG. 1: Examples of different local structures in the 3D FLS model and the crystal groundstates resulting when they are selected as the single FLS. In each case we plot the associated energy vs temperature curves for heating and cooling Monte Carlo runs (black lines) along with the equilibrium curve (red line). The latter is obtained by comparing the free energies of both the liquid and crystal, obtained by thermodynamic integration from $T=\infty$ and $T=0$, respectively. The step transition in energy is indicative of a first order transition. Also provided are the rotational degeneracy $g$, the groundstate energy $E_{o}$ and the crystal unit cell size (as a number of lattice sites), $Z$.

such that $\langle\vec{\phi}\rangle=0$ as $T \rightarrow \infty$. We now consider a high temperature expansion of the thermodynamic properties of the system. Let $S(\phi)$ denote the average entropy per site in a system restricted to this particular set of structure concentrations. To second order in $\phi$ we can write

$$
S(\vec{\phi})=S_{\infty}-\frac{1}{2} \vec{\phi}^{T} \cdot \hat{A} \cdot \vec{\phi}+O\left(\phi^{3}\right)
$$

such that the density of states in the structure space is approximated by a multi-dimensional Gaussian distribution,

$$
\Omega(\vec{\phi}) \approx \Omega_{\infty} \exp \left(-\frac{N}{2} \vec{\phi}^{T} \cdot \hat{A} \cdot \vec{\phi}\right)
$$

where $N$ is the number of lattice sites in the system. Note that $\Omega_{\infty}=2$ in this model (corresponding to the two spin states), and thus $S_{\infty}=\ln 2$. Since in the FLS model (as in many real world cases) the energy is completely determined by the numbers of different local structures, the 'interactions' in this model are entirely entropic and, in this high $T$ limit, encoded within the interaction matrix $\hat{A}$. The covariance matrix $\hat{C}$ of this distribution is given by $\hat{C}=\hat{A}^{-1}$.

Each instance of the model is characterised by specifying the energy $\epsilon_{i}$ for each local structure $i$. We can write the energy per site for a configuration described by a given structural concentration $\vec{c}$ as

$$
E(\vec{\phi})=\vec{\epsilon} \cdot \vec{c}=E_{\infty}+\vec{\epsilon} \cdot \vec{\phi}
$$

where $E_{\infty}=\vec{\epsilon} \cdot \vec{c}_{\infty}$. At a fixed temperature T, the free energy of the system with a given structural concentration $\vec{\phi}$ is

$$
\begin{aligned}
F(\vec{\phi}, T) & =E(\vec{\phi})-T S(\vec{\phi}) \\
& =E_{\infty}-T S_{\infty}+\vec{\epsilon} \cdot \vec{\phi}+\frac{T}{2} \vec{\phi}^{T} \cdot \hat{A} \cdot \vec{\phi}+O\left(\phi^{3}\right)
\end{aligned}
$$

The average concentrations at temperature $T$ satisfy the condition $\partial F / \partial \phi_{i}=0$ resulting in the following expression,

$$
\vec{\phi}(T)=-\frac{1}{T} \hat{C} \cdot \vec{\epsilon} .
$$

This simple expression gives an approximation of the temperature dependence of all local structures, which becomes exact in the infinite temperature limit. The concentration of local structure $i$ thus depends directly on the energy of FLS $j$ through the term $C_{i j} \epsilon_{j} / T$. At this level of approximation, all concentrations are proportional to $1 / T$. Substituting the equilibrium structural concentrations, we have

$$
\begin{aligned}
& E(T)=E_{\infty}-K / T \\
& S(T) \quad=S_{\infty}-\frac{K}{2 T^{2}} \\
& F(T)=E_{\infty}-T S_{\infty}-\frac{K}{2 T}
\end{aligned}
$$


where

$$
K=\vec{\epsilon}^{T} \cdot \hat{C} \cdot \vec{\epsilon}
$$

is the key quantity that connects the energy of structures to the thermodynamic properties of the liquid. Note that this quantity not only involves the structures energies, but also the entropic interaction between favoured structures. Using Eq. 10 to replace $T$ by $E$ we can write the microcanonical expression for the entropy,

$$
S(E)=S_{\infty}-\frac{\left(E-E_{\infty}\right)^{2}}{2 K}
$$

We note that a number of authors 21] have reported that the dependence of the configurational entropy on the inherent structure energy is well described by a quadratic function similar to that in Eq. 14.

To evaluate the quantity $K$ in the expressions above, we need the matrix elements of the covariance matrix $\hat{C}$. For a system of $N$ sites, the elements of $\hat{C}$ are related to the correlations between fluctuations of concentration of structures at infinite temperature through

$$
C_{i j}=N\left\langle\phi_{i} \phi_{j}\right\rangle_{\infty}
$$

Recalling that $\left\langle\phi_{i}\right\rangle_{\infty}=0$, a value of $C_{i j}>0$ corresponds to the case where concentrations of the two local structures correlate positively, due to a geometrical affinity for one another (i.e. the presence of one structure facilitates the formation of the other in its neighbourhood) while $C_{i j}<0$ indicates a geometric antipathy between structures $i$ and $j$. In this sense, the matrix $\hat{C}$ (or its inverse $\hat{A}$ ) encapsulates the essential constraints imposed by the local structures in space, at least at the level of the pairwise overlap. Note that the matrix $\hat{C}$ (unlike $K$ ) is independent of what energies are assigned to the local structures and so a single matrix applies for all possible versions of the FLS model.

In practice, we compute exactly the covariances in Eq. 15 by noting that it can be written as a finite sum of probabilities. Indeed, working on the value of the infinitetemperature correlation between concentrations $\left\langle c_{i} c_{j}\right\rangle_{\infty}$, we have:

$$
\begin{aligned}
\left\langle c_{i} c_{j}\right\rangle_{\infty} & =\left\langle\left(\frac{1}{N} \sum_{\overrightarrow{r_{1}}} \delta_{s\left(\overrightarrow{r_{1}}\right), i}\right)\left(\frac{1}{N} \sum_{\overrightarrow{r_{2}}} \delta_{s\left(\overrightarrow{r_{2}}\right), j}\right)\right\rangle_{\infty}(16) \\
& =\frac{1}{N^{2}} \sum_{\overrightarrow{r_{1}}} \sum_{\overrightarrow{r_{2}}}\left\langle\delta_{s\left(\overrightarrow{r_{1}}\right), i} \delta_{s\left(\overrightarrow{r_{2}}\right), j}\right\rangle_{\infty}
\end{aligned}
$$

where $\overrightarrow{r_{1}}$ and $\overrightarrow{r_{2}}$ are two lattice sites, and $\delta_{s\left(\overrightarrow{r_{1}}\right), i}$ equals one if the site at position $\overrightarrow{r_{1}}$ is in the local environment $i$, and zero otherwise. Using translation invariance, we can set $\overrightarrow{r_{1}}=\overrightarrow{0}$ and write:

$$
\left\langle c_{i} c_{j}\right\rangle_{\infty}=\frac{1}{N} \sum_{\vec{r}} \operatorname{Prob}\left(s_{\overrightarrow{0}}=i \& s_{\vec{r}}=j\right)
$$

where $\operatorname{Prob}\left(s_{\overrightarrow{0}}=i \& s_{\vec{r}}=j\right)$ denotes the joint probability that site $\overrightarrow{0}$ is in the local structure $i$ and site $\vec{r}$ is in $j$. If these two structures do not share any lattice site, they are independent at infinite temperature, and the probability thus factorizes to the average concentrations at infinite temperature $c_{\infty, i} c_{\infty, j}$. Going back to the shifted concentrations $\phi_{i}=c_{i}-c_{\infty, i}$, we can thus write:

$$
N\left\langle\phi_{i} \phi_{j}\right\rangle_{\infty}=\sum_{\vec{r} \sim \overrightarrow{0}} \operatorname{Prob}\left(s_{\overrightarrow{0}}=i \& s_{\vec{r}}=j\right)-c_{\infty, i} c_{\infty, j}
$$

where $\vec{r} \sim \overrightarrow{0}$ means that we consider only positions $\vec{r}$ whose local environment overlap with that of $\overrightarrow{0}$. We can rewrite this expression

$$
C_{i j}=c_{\infty, i} \sum_{\vec{r} \sim \overrightarrow{0}}\left(\operatorname{Prob}\left(s_{\vec{r}}=j \mid s_{\overrightarrow{0}}=i\right)-c_{\infty, j}\right)
$$

where $\operatorname{Prob}\left(s_{\vec{r}}=j \mid s_{\overrightarrow{0}}=i\right)$ denotes the conditional probability to find site $\vec{r}$ in local structure $j$, given that $\overrightarrow{0}$ is in $i$. This last expression is tractable, and can be computed by exact counting of all possible ways to successfully fit local structure $j$ at all positions overlapping with the origin. In practice, this required the evaluation of approximately $5 \times 10^{7}$ overlaps to compute the full covariance matrix in the $3 \mathrm{D}$ model.

The matrix $\hat{C}$ is singular, as the unphysical eigenvector $\phi_{\text {unphys }}=(1,1, \ldots 1)$, associated to concentrations that do not sum to unity, corresponds to a zero eigenvalue of $\hat{C}$. Surprisingly, we found that in the 3D FLS model, two other eigenvalues vanish. These non-trivial zero modes correspond to forbidden directions $\phi_{\text {forbidden }}$ in the concentration space: these compositions indeed incur an infinite entropy cost. Physically, they correspond to local structures whose neighbourhood must include some other type of structure: it is impossible to simultaneously increase their concentration, and decrease the concentration of all their "followers". We disregard these oddities in the following. To obtain the interaction matrix $\hat{A}$, we eliminate the zero modes by singular value decomposition, using the Moore-Penrose pseudo-inverse of $\hat{C}$.

In Fig. 2 we present a colour-coded representation of the covariance matrix. The local structures are indexed in blocks characterized by their number of up spins. So the all down local structure is given an index 0 , the local structure with a single up spin is structure 1 , the four structures with 2 up spins are structures 2 to 5 , arranged in order of increasing rotational multiplicity $g$, and so on. The gross features of $\hat{C}$, evident in Fig. 2, result from this choice of organizing the local structures. The blocks of positive covariance seen in the top left and bottom right corners correspond to the positive overlap of local structures that both consist of predominantly down or up spins, respectively. Similarly, the blocks of negative covariance in the top right and bottom left corners are the negative overlaps that result between a pair of structures whose majority spins are opposite in sign. The boundaries of these corner blocks correspond to the last of the 


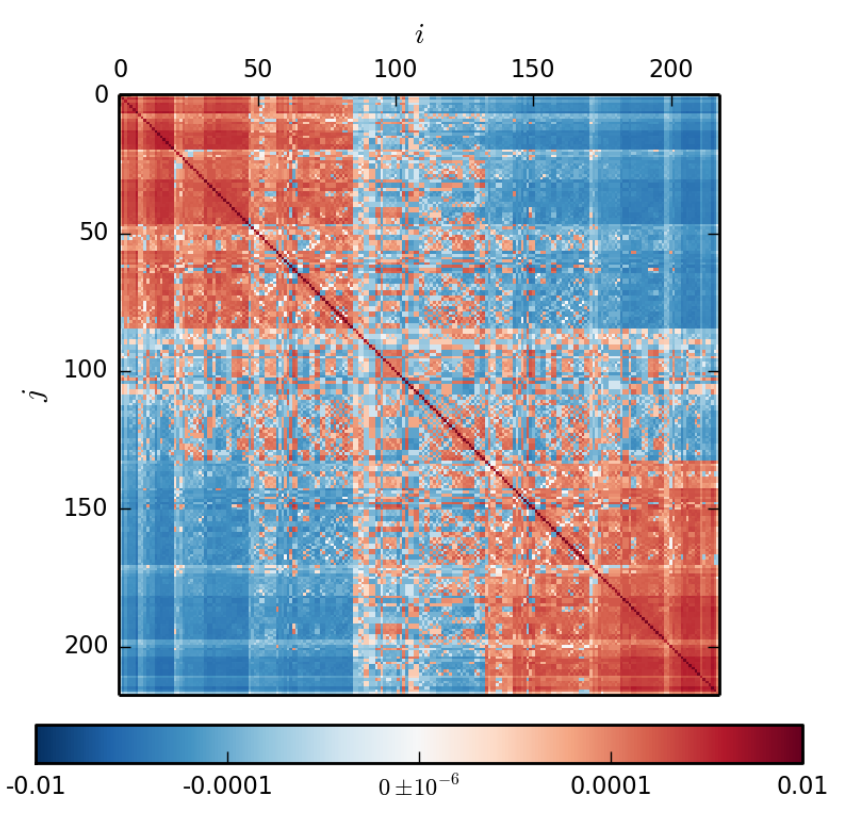

FIG. 2: A colour coded depiction of the values of the elements of the covariant matrix $\hat{C}$. Negative and positive elements are in shades of blue and red, respectively. These shades are log-scaled with a cut region around zero, as indicated by the colorbar. The local structures, listed by a numerical index running from 1 to 218 , are sorted by their number of up spins (hence the block structure of this matrix, as local structures with similar spin composition will tend to attract each other). Within each block, they are sorted with increasing geometrical multiplicity $g_{i}$.

5 up (or down) spin structures. The local structures with equal numbers of up and down spins make up the central cross-like feature with its complex patchwork of positive and negative covariance. The diagonal consists of the variances $N\left\langle\phi_{i}^{2}\right\rangle$ which must be positive.

To appreciate the detail of the covariance matrix we have extracted the covariances of just four local structures and presented them in Fig. 3. Clearly evident is the tendency for structures to have positive covariance when their majority spin is the same.

The block-like structure evident in $\hat{C}$ is largely absent in $\hat{A}$ as shown in Fig. 4. The interaction matrix exhibits a striking diagonal organization. Matrix elements close to the diagonal, i.e. corresponding to pairs of structures on slightly different from one another, we generally find negative values of $A_{i j}$ which imply structural compatibility. As we move further from the diagonal, the increasing difference in the sign of spins of the two local structures results in a positive (i.e. antagonistic) interaction. The diagonal values stand apart as they must be strongly positive to ensure that the high $T$ limit corresponds to a maximum in entropy.

How well does the high $T$ approximation fare in estimating properties of the FLS model? The freezing point $T_{f}$ is obtained by requiring that the free energy of the

\begin{tabular}{|c|c|c|c|c|}
\hline & $2.65 \mathrm{e}-04$ & $2.30 \mathrm{e}-04$ & $4.29 \mathrm{e}-06$ & $-3.29 \mathrm{e}-05$ \\
\hline & $2.30 \mathrm{e}-04$ & $8.85 \mathrm{e}-03$ & $3.32 \mathrm{e}-04$ & $-3.55 \mathrm{e}-04$ \\
\hline & $4.29 \mathrm{e}-06$ & $3.32 \mathrm{e}-04$ & $6.43 \mathrm{e}-03$ & $-3.43 \mathrm{e}-05$ \\
\hline & $-3.29 \mathrm{e}-05$ & $-3.55 \mathrm{e}-04$ & $-3.43 \mathrm{e}-05$ & $5.92 \mathrm{e}-03$ \\
\hline
\end{tabular}

FIG. 3: A selection of the covariances represented in Fig. 2. The numerical values of the covariances are provided in the matrix.

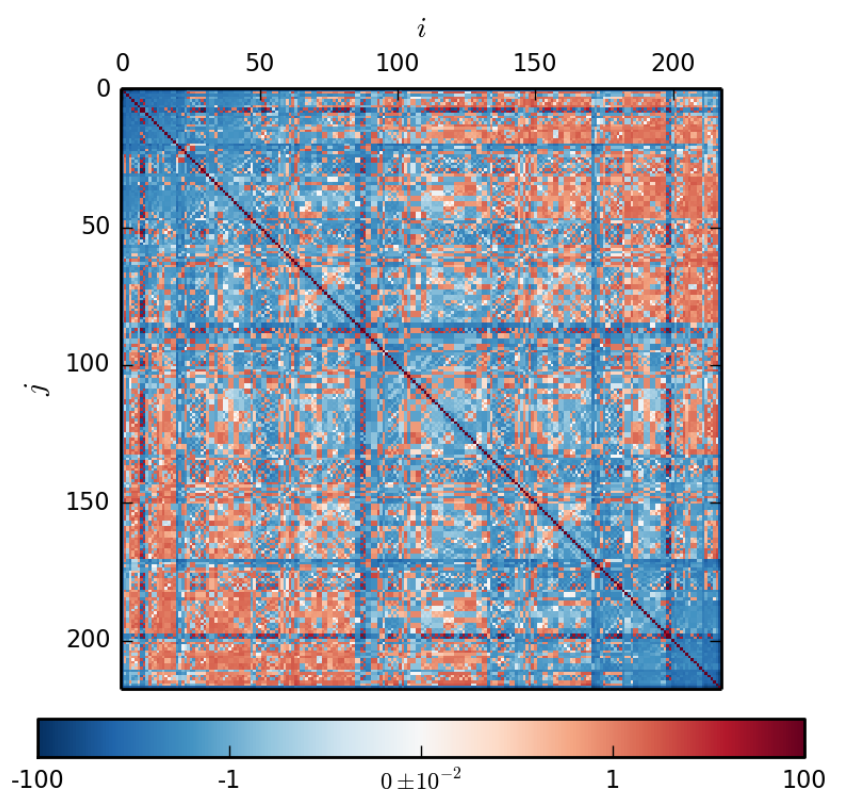

FIG. 4: A colour coded depiction of the values of the elements of the interaction matrix $\hat{A}$. Negative and positive elements are in shades of blue and red, respectively.

liquid, Eq. 12, is equal to the free energy of the crystal. The latter quantity can be reasonably approximated by zero entropy and a groundstate energy $E_{o}$. Doing so we arrive at the following expression for $T_{f}$,

$$
T_{f}=\left(\frac{E_{\infty}-E_{o}}{2 S_{\infty}}\right)\left(1+\sqrt{1-\frac{2 K S_{\infty}}{\left(E_{\infty}-E_{o}\right)^{2}}}\right)
$$

With the calculated covariances and the crystal ground- 
state energies we can use Eq. 21 to predict the melting points for various choices for favoured local structures and compare these predictions in Fig. 5 with those values obtained from the MC simulations. The approximate expression for $T_{f}$ provides an excellent prediction of the actual freezing point of a single FLS in the $3 \mathrm{D}$ and $2 \mathrm{D}$ models (with one exception in the latter case). This success is a reflection of the fact that liquids generally do not accumulate much local crystalline order before freezing, a direct consequence of the entropy cost of a single FLS. Small FLS concentrations are the basis for the expansion used to derive Eq. 21.

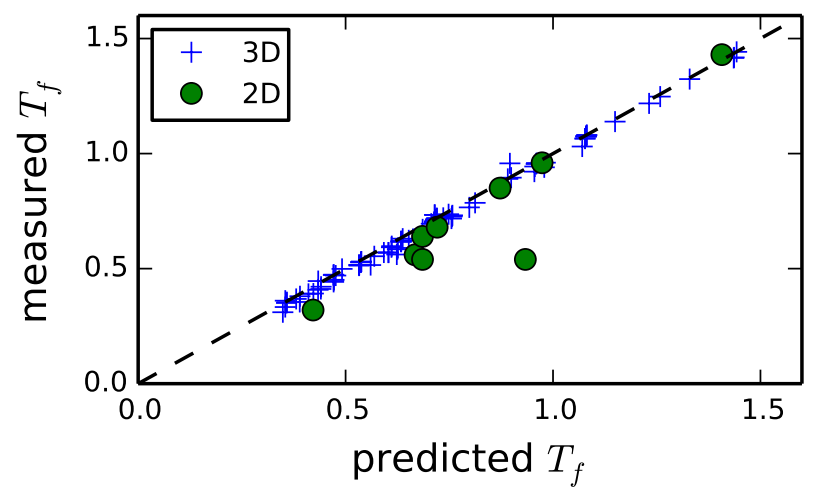

FIG. 5: A scatter plot of simulated melting points vs predictions from Eq. 21 for the single FLS cases in 3D and 2D. The dashed line corresponds to the case where the predicted and the actual values are equal.

More informative is the entropy as a function of energy. In Fig. 6 we compare the actual entropy as a function of energy, calculated using thermodynamic integration [16], with the approximate quadratic form in Eq. 14, for a variety of cases involving one, two or three FLS's. The key contributions of liquid structure to the stability (i.e. high entropy) of liquids are evident. Low symmetry FLS's and high overlap (of an FLS with itself and, in the case of multiple favoured structures, between FLS's) both contribute to larger $K$ 's and, hence, to higher liquid entropies for given energy. The high $T$ approximation performs reasonably for the case of a single FLS, consistent with the similar success for the approximate expression for $T_{f}$ discussed previously. We find that Eq. 14, while continuing to work at high energies, consistently underestimates the liquid entropy. This means that the pairwise interactions alone generally underestimate the capacity of structures to "pack" in space. Why this should be so is not obvious and represents an interesting question for future study.

Some attempts at simplifying the expression for the entropy are informative. Consider the following naive treatment of the problem in which we let each site be assigned one of the possible local structures independently of their neighbours. The result is a massive overestimation of the entropy as we have replaced the true number of possible states for a site, i.e. 2, with the very much larger number $2^{z}$ of possible local structures (with their rotational variants). The lesson here is that local structures inherently span over several degrees of freedom, and so they cannot decouple completely. We can however obtain a useful approximation by assuming that overlaps between structures are "neutral", i.e. that the presence of an FLS at a given site does not affect the probability of having an FLS at nearby sites. In this case, the entropy depends only on the symmetry properties of the local structures, which are encoded in the infinite-temperature concentration $c_{\infty, i}$ of FLS $i$ (Eq. 3). It is thus much simpler to compute: indeed, in this case, we neglect off-diagonal terms in the covariance matrix, and $K$ can be simply approximated as

$$
K \approx \sum_{i}^{N_{s}} \epsilon_{i}^{2} c_{\infty, i}
$$

The resulting approximation to $S(E)$, obtained by substituting this approximate $K$ into Eq. 14, has been plotted in Fig. 6. Eq. 22 provides an accurate estimation of $K$ when overlap between the FLS is small but clearly underestimates $K$ (i.e. underestimates the second order expression for $S(E)$ ) when the overlap is significant.

Finally, we note that $S(E)$ is a smoothly varying function of $E$ and, as such, can be reasonably described by Eq. 14 across much of the energy range if we allow $K$ to be an effective, adjustable parameter $K_{\text {eff fitted through }}$ the whole energy range. Capturing, as it does, the compounded complexities of local structure energies, the nonlinear contributions of overlap to the entropy and the influence of multiple FLS's, $K_{\text {eff }}$ should provide a useful quantity in the comparison of the role of local structure in different liquids, with the drawback that it is no longer a coefficient in an exact expansion, and thus cannot be computed exactly from simple rules.

\section{THE SUSCEPTIBILITY OF A LIQUID TO FLUCTUATIONS IN LOCAL STRUCTURE}

Structure in liquids matters most when we are interested in how that liquid will respond to some perturbation. A crystal surface, for example, will grow if the perturbations in local crystal order that it induces in the adjacent liquid are unstable and result in the propagation of the interface. Crystal nucleation theory seeks to describe the unstable wing of the (metastable) equilibrium distribution of the local structures in the supercooled liquid. Structural fluctuations in liquids involving more than pairwise correlations have become directly observable with techniques such as fluctuation electron microscopy 22]. To consider fluctuations in structure we need to generalize our treatment of the free energy to include spatial heterogeneities in structure, i.e. $\vec{\phi}(\vec{r})$. The energy expression is easily generalized,

$$
E[\vec{\phi}]=E_{\infty}+\frac{1}{N} \sum_{\vec{r}} \vec{\phi}(\vec{r}) \cdot \vec{\epsilon}
$$



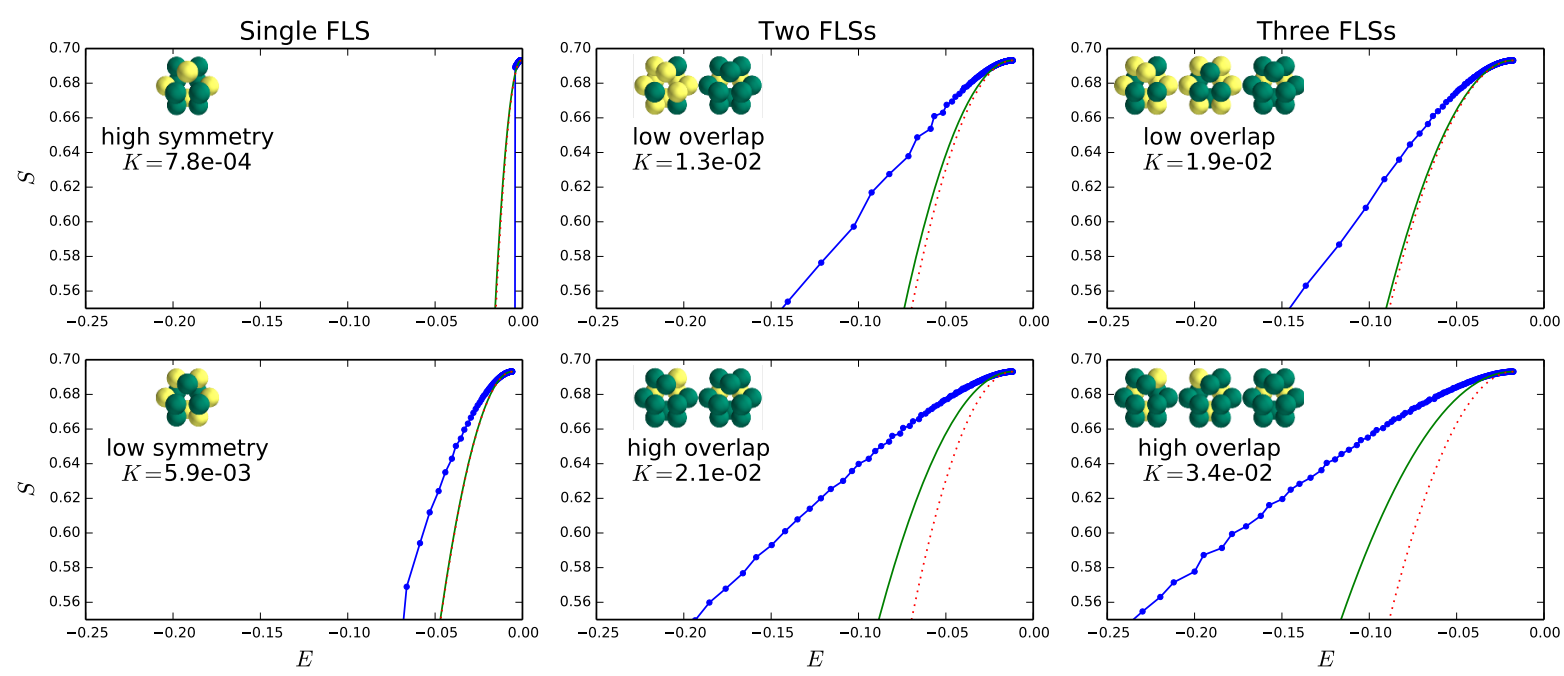

FIG. 6: The liquid entropy as a function of energy calculated by thermodynamic integration from simulations (blue circles) and using the approximate expression in Eq. 14 using the exact expression for $K$ (Eq. 13 ) (green line) and the approximate $K$ (Eq. 22 (red dots). Examples with one, two and three FLS's are presented along with the value of $K$ from Eq. 13

where $N$ is the number of sites. For the entropy, we shall again resort to our high $T$ (and, hence, small $\phi$ ) expansion. Now $S[\vec{\phi}]$ is a functional of $\vec{\phi}(\vec{r})$ and so the Taylor expansion is of the form

$$
S[\vec{\phi}] \approx S_{\infty}-\frac{1}{2 N^{2}} \sum_{\vec{r}_{1}} \sum_{\vec{r}_{2}} \vec{\phi}^{T}\left(\vec{r}_{1}\right) \cdot \hat{A}\left(\vec{r}_{1}-\vec{r}_{2}\right) \cdot \vec{\phi}\left(\vec{r}_{2}\right)
$$

and the equilibrium condition is

$$
N \frac{\delta F}{\delta \vec{\phi}\left(\vec{r}_{1}\right)}=\vec{\epsilon}+\frac{T}{N} \sum_{\vec{r}_{2}} \hat{A}\left(\vec{r}_{12}\right) \cdot \vec{\phi}\left(\vec{r}_{2}\right)=0
$$

The spatial dependence of the interaction matrix $\hat{A}$ elements provides the contains the essential information about the locality of the overlap of local coordination shells. The spatially resolved covariance matrix $\hat{C}(\vec{r})$ is given by

$$
C_{i j}(\vec{r})=N\left\langle\phi_{i}(0) \phi_{j}(\vec{r})\right\rangle
$$

and corresponds to the two-point correlation between local structures. In the high $T$ limit, these structural correlation functions can be calculated by an extension of the approach used to produce Fig. 2. This analysis is left for future work. The connection between the covariance matrix $\hat{C}\left(\vec{r}_{1}, \vec{r}_{2}\right)$ and the entropy via the interaction matrix $\hat{A}\left(\vec{r}_{1}, \vec{r}_{2}\right)$ is not as straightforward as it is for the homogeneous case since the relation between the two matrices will involve some sort of nonlocal convolution. Allowing for this complexity, we can make the following observation. The spatial correlations of the covariance
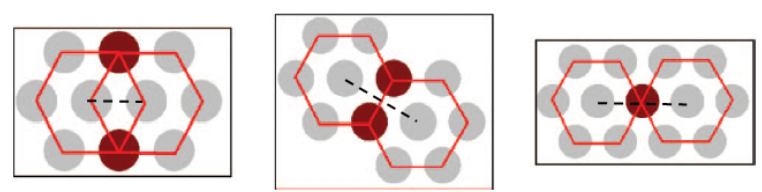

FIG. 7: The extent of overlap between a pair of local structures in the $2 \mathrm{D}$ triangular lattice. The dashed line indicates the three separations between sites for which the structure overlap is non-zero.

matrix $\hat{C}(\vec{r})$ are restricted to the short distance set by the extent of overlap of local structures. In Fig. 7 we show how the overlap of local coordination shells in the $2 \mathrm{D}$ triangular lattice only extends out to second nearest neighbour sites. A similar length is obtained in the fcc lattice in 3D. For separations beyond this overlap limit, $C_{i j}(\vec{r})=0$. While we expect that the interaction matrix $\hat{A}(\vec{r})$ will exhibit a similar spatial extent of its influence, the exact relation must wait establishing a clearer picture of the relation between the entropic interactions between local structures and their spatial correlations.

\section{DISCUSSION}

We have presented a formalism in which the free energy of a liquid is expressed explicitly in terms of the local coordination structure. The novel feature of this theory is the treatment of the structural contribution to the entropy which allows us to describe the entropic consequences of the geometry of an individual favoured local structure and the impact of multiple favoured local 
structures. The connection between structure and entropy arises from the overlap of the local coordination shells and the degree of local structural constraint this imposes. While we have restricted ourselves to the high $T$ approximation, the overlap terms are athermal and so much of the details of the affinities between pairs of local structures is retained in the interaction matrix $\hat{A}$ and its inverse, the covariance matrix $\hat{C}$. We considered only the expansion of the entropy to quadratic order in the energy. In the context of single FLS liquids, we previously described how higher order terms in this expansion can be computed as sums of overlaps of larger clusters [17. However, we found that the complexity of these terms increases very rapidly, for only moderate improvement of the entropy estimate (data not shown).
The extension of the formalism to spatial inhomogeneities in the field of structural concentrations has been introduced. This spatial variation, if coupled to a free energy with terms of order greater than the quadratic ones that characterise our high $T$ approximation, will provide the basis for a theory of the crystal-liquid interfacial free energy and the free energy of crystal nuclei in which, for the first time, the influence of non-crystalline local structures can be explicitly mapped out.

Acknowledgements $\mathrm{PH}$ acknowledges the financial support of the Australian Research Council. PR is supported by "Initiative Doctorale Interdisciplinaire 2013" from IDEX Paris-Saclay. Figures realized with Matplotlib 23] and Mayavi2 24].
[1] J. L. C. Daams and P. Villars, J. Alloys Comp. 252, 110 (1997).

[2] J.W. Steed, CrystEngComm.5, 169 (2003).

[3] J. D. Bernal, Nature 183, 141 (1959); A. Rahman, J. Chem. Phys. 45, 2585 (1966); J. L. Finney, Proc. Roy, Soc. A 319479 (1970).

[4] T. Ogawa and M. Tanemura, Proc. Theor. Phys. 51, 399 (1974); M. Tanemura, Y. Hiwatari, H. Matsuda, T. Ogawa, N. Ogita and A. Ueda, Prog. Theor. Phys. 58, 1079 (1977).

[5] H. Jónsson and H. C. Andersen, Phys. Rev. Lett. 602295 (1988).

[6] Y. H. Chui, R. J. Rees, I. K. Snook, B. OMalley and S. P. Russo, J. Chem. Phys. 125, 114703 (2006).

[7] J. P. K. Doye and D. J. Wales, Phys. Rev. Lett. 86, 5719 (2001).

[8] C. P. Royall and S. R. William, Phys. Rep. 560, 1 (2015); Y. Q. Cheng and E. Ma, Prog. Mater. Sci. 56, 379 (2011).

[9] F. C. Frank and J. S. Kasper, Acta Crystallogr. 11, 184 (1958).

[10] I. Procaccia, Eur. Phys. J. Special Topics 178, 81 (2009).

[11] H. G. E. Hentschel, V. Ilyin, N. Makedonska, I. Procaccia and N. Schupper, Phys. Rev. E 75, 050404 (2007)

[12] L. Boué, E. Lerner, I. Procaccia and J. Zylberg, J. Stat.
Mech. P11010 (2009).

[13] L. Boué, H. G. E. Hentschel, V. Ilyin and I. Procaccia, J. Phys. Chem. B 115, 14301 (2011).

[14] I. Procaccia and I. Regev, Europhys. Lett. 97, 36010 (2012)

[15] P. Ronceray and P. Harrowell, Europhys. Lett. 96, 30065 (2011).

[16] P. Ronceray and P. Harrowell, J. Chem. Phys. 136, 134504 (2012).

[17] P. Ronceray and P. Harrowell, Phys. Rev. E, 87, 053313 (2013).

[18] P. Ronceray and P. Harrowell, Phys. Rev. Lett. 112, 017801 (2014).

[19] P. Ronceray and P. Harrowell, Soft Matt. 11, 3322 (2015).

[20] A. B. Bortz, et al J. Comp. Phys. 17, 10 (1975).

[21] S. Sastry, J. Phys.: Cond. Matt. 12, 6515 (2000); A. Heuer, J. Phys.: Cond. Matt. 20, 373101 (2008).

[22] J. M. Gibson, M. M. J. Treacy and P.M. Voyles, Ultramicroscopy 83, 169 (2000).

[23] J. D. Hunter, Comput. Sci. Eng., 9 (3), 90-95 (2007).

[24] P. Ramachandran and G. Varoquaux, Comput. Sci. Eng. 13, 40 (2011). 